\title{
PROCEDIMIENTOS PARA HACER EFECTIVO EL DERECHO DE RÉPLICA
}

Miguel Ángel RODRÍGUEZ VÁZQUEZ*

\section{INTRODUCCIÓN}

Del análisis histórico del sistema jurídico mexicano podemos darnos cuenta que "el tiempo no ha sido un buen aliado del derecho de réplica", pues quienes han tenido la responsabilidad de reconocerlo en el ámbito constitucional y regularlo legalmente no lo hicieron con puntualidad. Tuvieron que pasar alrededor de noventa años para que ello sucediera, desde que entró en vigor la Constitución mexicana de 1917 y hasta que se consagró en la misma en 2007. Después pasaron otros ocho años para que en 2015 se expidiera la Ley Reglamentaria del Artículo 6o., párrafo primero, de la Constitución Política de los Estados Unidos Mexicanos, en materia del Derecho de Réplica.

Pero la metáfora del tiempo en esta materia también aplica para analizar los plazos y términos consagrados en los procedimientos extrajudicial y judicial que se deben cumplir para hacer efectivo el derecho de réplica. Advertimos consecuencias desfavorables que se pueden generar para las personas que se hayan visto afectadas por información inexacta o agraviante publicada o transmitida por los sujetos obligados a garantizar el mencionado derecho. En particular, nos preocupa el diseño normativo de los plazos en casos de que haya necesidad de llegar hasta la emisión de una sentencia en el juicio de amparo respectivo para garantizar el derecho de réplica.

Doctor en derecho por la Universidad Nacional Autónoma de México; profesorinvestigador en la Universidad Juárez del Estado de Durango; investigador nivel I del Sistema Nacional de Investigadores; miembro asociado de la Sección Mexicana del Instituto Iberoamericano de Derecho Constitucional; editorialista de El Sol de Durango, y magistrado en retiro,drma_rodriguez@hotmail.com.

Fecha de recepción: 30 de mayo de 2016.

Fecha de dictamen: 14 de septiembre de 2016. 
Este trabajo tiene como objetivo demostrar, a través del análisis de las disposiciones jurídicas vigentes y bajo una perspectiva de derechos humanos histórica y comparada, que el tiempo consagrado en los procedimientos para hacer efectivo el derecho de réplica corre en contra del solicitante por los agravios que se pueden producir por la violación de alguno o algunos de sus derechos de la personalidad y las repercusiones económicas o políticas, si se llegara en el judicial hasta el término de la segunda instancia y, posteriormente, hasta la emisión de la sentencia en amparo.

Es así que en este texto, en primer lugar, se aborda la parte conceptual para dejar en claro la naturaleza jurídica del derecho de réplica como un - derecho humano, tal y como es reconocido por los tratados internacionales

- de los cuales el Estado mexicano forma parte, así como por nuestra carta magna. Después se analiza, desde el punto de vista histórico, el desarrollo normativo hasta consagrarse explícitamente como derecho humano en el ámbito constitucional. En la parte central del trabajo se estudia la Ley Reglamentaria del Artículo 6o., párrafo primero, de la Constitución Política de los Estados Unidos Mexicanos, en materia del Derecho de Réplica, así como el Código Federal de Procedimientos Civiles, para identificar el tiempo que tiene que transcurrir en el proceso judicial y los problemas que ello representa para que se haga efectivo tal derecho. Finalmente, se formulan conclusiones que se derivan de lo abordado en el cuerpo de este trabajo.

\section{Delimitación conceptual del derecho de RÉplica}

En primer lugar, conviene anotar que el derecho al que nos estamos refiriendo lo llamamos "derecho de réplica", por así citarlo el artículo 6o. de la Constitución mexicana. En el sistema interamericano y las Constituciones de los Estados a los que se hará mención en este trabajo, este derecho toma otras denominaciones, lo que se debe a diversas razones, como "a la calidad de los sujetos principalmente porque sean funcionarios como en el caso de Francia o actores en la oposición y las minorías como en el caso colombiano..." (Rosas, 2011: 99). Incluso, hay quien diferencia entre rectificación y réplica, como Ekmekdjian, quien explica que la primera se ejerce cuando existe información errónea sobre algún aspecto de la persona, en tanto que la segunda, "cuando hay un ataque malicioso contra la honra o aspectos fundamentales de la persona o sus creencias” (Ekmekdjian, 1996: 66).

Como sabemos, en el artículo 14 de la Convención Americana sobre Derechos Humanos se reconoce el derecho de rectificación o respuesta que tiene toda persona por informaciones inexactas o agraviantes emitidas 
en su perjuicio a través de medios de difusión legalmente reglamentados y que se dirijan al público en general, en los términos que establezca la ley. En el artículo 20 de la Constitución de Colombia se hace mención del derecho de rectificación en condiciones de equidad, y en el artículo 112 a los partidos políticos se les garantiza su derecho a la réplica. En Argentina, como no se ha reconocido explícitamente en su Constitución, se recurrió al artículo 14.1 de la Convención Americana sobre Derechos Humanos, y se estableció el derecho por Ley 23054 ratificada el 5 de septiembre de 1984, en donde se emplean los términos de rectificación o respuesta utilizados por la propia Convención.

Ahora bien, con independencia de su denominación, se concibe al derecho de réplica como un derecho humano, tal como señala la Corte Interamericana de Derechos Humanos (OC-7/86), en el sentido de que "es un derecho al cual son aplicables las obligaciones de los Estados Partes consagradas en los artículos 1.1 y 1.2 de la Convención”. Parte de la doctrina se ha pronunciado en ese sentido; por ejemplo, Miguel Carbonell considera que es "el derecho que tiene toda persona ofendida o injustamente aludida por algún medio de comunicación...” (Carbonell, 2011: 446).

No obstante, hay quien afirma lo contrario, como Ghersi, quien manifiesta que "es un remedio autónomo, específico, con características especiales, con una faz judicial y una faz extrajudicial, pero que evidentemente no es un derecho en sí mismo" (2000: 86). Por su parte, Cifuentes expresa que "no es un derecho subjetivo ni uno de los personalísimos. Es un procedimiento de tutela particular judicial o extrajudicial, cuyo objeto es la reposición del derecho violado" (1998: 631).

Nos afiliamos a la tesis del máximo órgano jurisdiccional del sistema interamericano de derechos humanos, en el sentido de que sí es un derecho, que se corresponde con la libertad de pensamiento y de expresión: "la ubicación del derecho de rectificación o respuesta (artículo 14) inmediatamente después de la libertad de pensamiento y expresión (artículo 13), confirma esta interpretación” en la Convención Americana (OC-7/86). Asimismo, en la Constitución mexicana en el párrafo primero del artículo 6o. se reconoce como derecho humano. A lo anterior agregamos que no es un límite a la libertad de expresión, "sino el reconocimiento de otro de igual rango, que es inherente al legítimo derecho de la personalidad" (Pierini, 1998: 205).

Desde el punto de vista legal, la fracción II del artículo 2o. de la Ley Reglamentaria del Artículo 6o., párrafo primero, de la Constitución Política de los Estados Unidos Mexicanos, en materia del Derecho de Réplica consagra que se entenderá por "derecho de réplica" lo siguiente: 
Esta revista forma parte del acervo de la Biblioteca Jurídica Virtual del Instituto de Investigaciones Jurídicas de la UNAM

El derecho de toda persona a que sean publicadas o difundidas las aclaraciones que resulten pertinentes, respecto a datos o informaciones transmitidas o publicadas por los sujetos obligados, relacionados con hechos que le aludan, que sean inexactos o falsos, cuya divulgación le cause un agravio ya sea político, económico, en su honor, vida privada y/o imagen.

De lo antes expuesto, con independencia de la definición legal, consideramos que es un derecho que tiene toda persona cuando se sienta perjudicada por una información inexacta o agraviante que se hizo pública a través de un órgano de difusión, a contradecirla por no ser cierta o a argüir - en caso de serlo, a través de la publicación o transmisión de lo que mani- fieste al respecto, de manera gratuita y en condiciones de equidad por el mismo medio que se divulgó.

En tal definición, consideramos que la esencia del derecho de rectificación es la idea de la "contradicción", que según el Diccionario de la lengua española significa "probar que algo no es cierto o correcto..." (comprende la falsedad). De ahí que se trate de contradecir una versión publicada o transmitida por un órgano de difusión y presentar la propia (que se considera cierta y correcta, por eso se rectifica) para defender la honra, vida privada y/o imagen ante el público en general. Asimismo, un significado de la palabra "rectificación" es, precisamente, "contradecir a alguien en lo dicho, por considerarlo erróneo", y la palabra "respuesta" también puede significar "réplica, refutación o contradicción"; además, la palabra "réplica" significa "respuesta". Sin embargo, reconocemos que puede acontecer que se difunda públicamente una información cierta, pero que sea agraviante porque viole, por ejemplo, el derecho a la intimidad, entonces se trata de replicar, es decir, de "argüir", de dar una explicación o exponer sus razones, "aducir o alegar una razón o un argumento a favor o en contra de alguien o algo", lo cual forma parte también del derecho de réplica.

Con base en ese criterio es que se considera que en la Ley del Derecho de Réplica existe cierta confusión en el uso de los términos, pues de su análisis íntegro se advierte que en algunos de sus artículos se presenta esta cuestión, como es el caso del 6o., que en el párrafo primero dice "rectificación o respuesta", en tanto que en el último párrafo se refiere a "la réplica, rectificación o respuesta”. En tal virtud, es enriquecedor analizar doctrinalmente esos conceptos y, por supuesto, también resultará interesante conocer la interpretación que — en su momento - llegue a realizar la Suprema Corte de Justicia de la Nación. 


\section{ANTECEDENTES CONSTITUCIONALES Y LEGALES}

DEL DERECHO DE RÉPLICA

De la lectura del texto constitucional original de 1917 en la parte relativa a "Garantías individuales" advertimos que el derecho de réplica no estuvo reconocido explícitamente. No obstante, se consagró en la Ley sobre Delitos de Imprenta, del 9 de abril de 1917, expedida por Venustiano Carranza en su carácter de Primer Jefe del Ejército Constitucionalista, con la justificación siguiente: "entretanto el Congreso de la Unión reglamenta los artículos 6o. y 7o. de la Constitución General de la República”.

Dicha normativa ha sido criticada por juristas como Jorge Islas e Ignacio Burgoa. El primero dice: "ya que además de haber sido promulgada días antes que nuestra actual Constitución, fue creada por unas cuestionables facultades del Primer Jefe del Ejército Constitucionalista Venustiano Carranza" (Islas, 2002: 81). De tal manera que pone en tela de duda su constitucionalidad al haberse emitido en la fecha mencionada y ser publicada en el Diario Oficial de la Federación el 12 de abril de 1917. Sin embargo, nuestra carta magna estuvo vigente a partir del 1o. de mayo de ese año, por lo que estaba reglamentando los artículos 6o. y 7o. que aún no se encontraban en vigor. Además, técnicamente, para que una ley expedida en tales circunstancias esté vigente, dice el segundo de los juristas, es menester que la Constitución "la incorpore a su normación, o declare su subsistencia o faculte para declararla" (Burgoa, 1982: 360), lo cual no se hizo.

No obstante, la Suprema Corte de Justicia de la Nación en diversas tesis defendió su vigencia y daremos dos ejemplos al respecto. El primero consiste en la tesis aislada que emitió la Primera Sala, cuyo rubro es "LeY DE Imprenta De 9 de AbRIL DE 1917” (Tesis s/n), en la que se contiene una afirmación sustentada en argumentos muy endebles, en el sentido de que la legislación preconstitucional, principalmente la Ley de Imprenta, "tiene fuerza legal y deben ser aplicadas en tanto que no pugne con la Constitución, o sean especialmente derogadas". En otra tesis aislada cuyo rubro es "Ley de Imprenta" (Tesis s/n), la Primera Sala aduce que ya se había promulgado la Constitución; por tanto, la expedición de la ley "hubiera carecido de objeto, si sólo se hubiera dado para que estuviera en vigor por el perentorio término de 17 días". Aunado a lo anterior, se había aclarado que estaría en vigor mientras el Congreso de la Unión hiciera la reglamentación de los artículos 6o. y 7o. constitucionales, "y como no se ha derogado ni reformado dicha Ley de Imprenta, ni se ha expedido otra, es indudable que debe estimarse en todo su vigor". Sin embargo, no se refiere a los pro- 
blemas de técnica constitucional a los que hemos aludido, de ahí que se coincida en que era dudosa su constitucionalidad.

Tuvieron que pasar décadas para que el Reglamento de la Ley Federal de Radio y Televisión en materia de Concesiones, Permisos y Contenido de las Transmisiones de Radio y Televisión, del 10 de febrero de 2002, regulara el derecho de réplica en una de sus disposiciones, concretamente en el artículo 38, párrafo primero. En cuanto a su contenido, era "sumamente pobre, limitado, caprichoso y confuso" (Esteinou, 2003: 170). Una de las críticas consistió en que uno de los supuestos para que procediera la réplica es que no se hubiera citado la fuente de información, por lo que una interpretación a contrario sería que si se citara la fuente, entonces ¿se podría vulnerar el derecho al honor, vida privada y/o imagen?

Posteriormente, el Código Federal de Instituciones y Procedimientos Electorales, en su artículo 233, párrafos tercero y cuarto, también se refirió al derecho de réplica, y lo consagró a favor de los partidos políticos, los precandidatos y los candidatos, mas aclaraba que se ejercería "en la forma y términos que determine la ley de la materia", la cual, de acuerdo con su artículo décimo transitorio, se debería expedir "a más tardar el 30 de abril de 2008" por el Congreso de la Unión, a lo que no se dio cumplimiento.

Por tal omisión legislativa, el Tribunal Electoral del Poder Judicial de la Federación se vio obligado a fijar criterios. En dicha jurisdicción federal se resolvieron diversos asuntos sobre el derecho de réplica. Nos referiremos al resuelto por la Sala Superior del Tribunal Electoral del Poder Judicial de la Federación y que está contenido en el expediente SUP-RAP-175/2009, en el que se determinó el procedimiento para cumplir de manera pronta y que no se siguieran vulnerando derechos, además de que no se causaran daños irreparables, desde el punto de vista electoral, a quienes interpusieron el recurso de apelación.

Se trata de la impugnación realizada por el presidente del secretariado estatal del Partido de la Revolución Democrática en Nuevo León y el candidato a diputado federal en el distrito 8, en contra de la resolución CG276/2009 del Consejo General del Instituto Federal Electoral, respecto de la denuncia presentada en contra de la editorial el Sol, S. A. de C. V., con nombre comercial "El Norte", por hechos que se consideraron que constituían infracciones al Código Federal de Instituciones y Procedimientos Electorales. En esa resolución se determinó desechar la queja interpuesta, con el argumento de que la nota periodística no constituía un acto en materia electoral que pudiera afectar el debido desarrollo del proceso electoral y a su resultado, y menos que contraviniera los principios constitucionales que reviste toda elección. En contra de tal resolución se 
interpuso el recurso de apelación, el que fue procedente y fundado, por lo que la Sala Superior determinó revocar el acuerdo del Consejo General del Instituto Federal Electoral.

Conviene resaltar que se determinó que el procedimiento especial sancionador era el adecuado, en virtud de que la materia electoral necesita ser resuelta con rapidez, pues de no ser así, "no tendría los mismos efectos en los electores". El procedimiento sancionador ordinario para su terminación requería alrededor de sesenta y cuatro a ciento veintinueve días, mientras que el procedimiento especial sancionador tan sólo cinco a seis días, aproximadamente. Con base en los criterios utilizados en la sentencia, se estimó erróneo considerar que no era un acto en materia electoral, ya que la nota se emitió en periodo de campaña, y que no se trataba únicamente de una opinión, sino de una relación de hechos, por lo que se determinó que no era procedente desechar y, por ello, se revocó el acuerdo del Consejo General del Instituto Federal Electoral.

Por su parte, la Suprema Corte también fijó criterios importantes en la parte sustantiva; por ejemplo, en el amparo directo 6/2009 se emitió la tesis cuyo rubro es "Derecho a la intimidad. El derecho de RÉplica no REPARA LA INTROMISIÓN EN AQUEL” (Tesis 1a. XLIV/2010), y en el amparo directo 28/2010 se fijó la tesis cuyo rubro es "LiberTAD DE eXPREsión. Su FUNCIONAMIENTO EN CASOS DE DEBATE PERIODÍSTICO ENTRE DOS MEDIOS DE COMUNicACión" (Tesis 1a. XXVI/2011 [10a.]).

Fue hasta el 4 de noviembre de 2015 cuando se publicó en el Diario Oficial de la Federación la Ley Reglamentaria del Artículo 6o., párrafo primero, de la Constitución Política de los Estados Unidos Mexicanos, en materia del Derecho de Réplica. En las iniciativas presentadas (catorce desde 2007) se abordaban diversos temas, pero en lo general se hacía mención de la necesidad de que se tramitara de manera rápida. Algunas sí fueron muy específicas en regular tanto el procedimiento extrajudicial y parte del judicial ante la autoridad federal, tal como lo analizaremos en el siguiente apartado, en el que pretendemos demostrar que si se tuvieran que agotar tales procedimientos, se podrían generar consecuencias desfavorables para el agraviado.

\section{ANÁLisis de LOS PRocedimientos EXTRAJUdicial y JUdicial}

Ahora bien, ya centrándonos en el procedimiento que consagra la Ley Reglamentaria del Artículo 6o., párrafo primero, de la Constitución Política de los Estados Unidos Mexicanos, en materia del Derecho de Réplica, pu- 
blicada en el Diario Oficial de la Federación el 4 de noviembre de 2015, encontramos que desde que se llevó a cabo la discusión en el Congreso de la Unión fue objeto de críticas. Una de ellas la formuló Vladimir Chorny, quien dijo que aun sin apelación se "traduce en un procedimiento complejo, engorroso, donde obligan a la persona afectada a probar la falsedad de la información...". También consideró que en el terreno político electoral "el daño a una campaña o candidata puede ser irreparable si no se corrige o contrasta la información en los muy pocos días siguientes a su difusión”, y tratándose del resto de las personas, "su imagen puede ser dañada y sufrir criminalización, exclusión y rechazo. La inmediatez acá es indispensable"

- (2015: 3). En tal virtud, procederemos a analizar los tiempos establecidos - para percatarnos de cuánto puede tardar la reparación de los derechos vulnerados por información inexacta o agraviante.

Cuando se trate de transmisiones en vivo, si el formato del programa lo permite y a juicio del medio de comunicación es procedente la solicitud presentada, se realizará la rectificación o respuesta durante la misma transmisión, por lo que en este caso no habrá problema con el tiempo en que se ejerce el derecho aludido. Pero si no se trata de transmisiones en vivo, el escrito se deberá presentar ante el sujeto obligado en un plazo no mayor a cinco días hábiles (deberían ser más días a nuestro juicio, porque se parte de la difusión de la información, mas no de su conocimiento por el afectado), contados a partir del día siguiente al de la publicación o transmisión de la información que se desea rectificar o responder (artículo 10). De ese momento, el sujeto obligado tendrá un plazo máximo de tres días hábiles para resolver sobre la procedencia de la solicitud de réplica, luego tres días hábiles más para notificar al promovente su decisión (artículo 11).

En caso de que se considere que es procedente la solicitud, deberá publicarse o transmitirse al día hábil siguiente al de la notificación de la resolución si es que se trata de programas o publicaciones de emisión diaria, y en los demás casos, en la siguiente transmisión o edición (artículo 14). Cuando se trata de agencias de noticias deberán difundir la rectificación o respuesta en un plazo máximo de veinticuatro horas, contadas a partir de la fecha en que se resuelva la procedencia de la solicitud de réplica; entonces, el medio de comunicación deberá publicar o transmitir la réplica o rectificación al día hábil siguiente al de la notificación de las agencias o productores independientes (artículo 17).

Todo lo anterior acontece cuando el sujeto obligado considere que es procedente la solicitud de réplica; no obstante, ¿qué sucede si rechaza la solicitud? La Ley que se analiza establece ocho supuestos en los que el sujeto obligado puede negarse a llevar a cabo la publicación o transmisión 
(artículo 19). Sin embargo, si el afectado no está de acuerdo con la decisión por estimar que no se encuentra justificada, podrá presentar solicitud de inicio del procedimiento judicial ante el juez de distrito competente dentro de los cinco días hábiles, de conformidad con las reglas previstas legalmente (artículo 24).

Una vez que el juez de distrito admita la solicitud, mandará a emplazar en forma inmediata al sujeto obligado, para que dentro de los cuatro días hábiles siguientes al que surta sus efectos el emplazamiento, produzca su contestación por escrito y haga valer sus excepciones y defensas que estime pertinentes (artículo 30). Cabe aclarar que en el procedimiento no se sustanciarán incidentes de previo y especial pronunciamiento, y en caso de que si se presentaran, se resolverán al emitirse la resolución que ponga fin al procedimiento (artículo 31).

Dentro de los dos días hábiles siguientes a que se haya presentado la contestación de la demanda, o, en su caso, concluido el término legal para hacerlo, el juez citará a las partes a audiencia de desahogo de pruebas y alegatos, dentro de la cual podrá dictar sentencia o, en su defecto, dentro de las veinticuatro horas (artículo 34).

Vamos a ilustrar lo antes dicho para un mayor entendimiento:

\section{Procedimiento extrajudicial}

I. Transmisiones en vivo: la réplica se ejerce en la misma transmisión.

II. Transmisiones que no son en vivo:

1. Presentación del escrito: cinco días hábiles (a partir del día siguiente al de la publicación o transmisión).

2. Resolución sobre procedencia: tres días hábiles de la recepción de la solicitud.

3. Notificación de la resolución: tres días hábiles a partir de emitir resolución.

4. Publicación o transmisión de la aclaración: al siguiente día hábil al de la notificación.

\section{Procedimiento judicial ante EL Juez de distrito}

1. Solicitud de inicio: cinco días hábiles siguientes.

2. Emplazamiento: cuatro días hábiles siguientes al que surta efecto.

3. Citación a audiencia de pruebas y alegatos: dos días hábiles siguientes.

4. Sentencia: en la misma audiencia o veinticuatro horas después.

Hasta aquí, por los tiempos señalados, advertimos que se sustancian de una manera rápida los procedimientos extrajudicial y judicial para ha- 
Esta revista forma parte del acervo de la Biblioteca Jurídica Virtual del Instituto de Investigaciones Jurídicas de la UNAM

cer valer el derecho de réplica. Sin embargo, hay que tener presente que en contra de las resoluciones que emita el juez de distrito procede el recurso de apelación (artículo 35), en términos de lo previsto por el Código Federal de Procedimientos Civiles (en adelante CFPC) (Diario Oficial de la Federación del 24 de febrero de 1943).

Llegado el caso de que se interpusiera el recurso de apelación y des94 pués se promoviera el juicio de amparo, el procedimiento se alargaría con el consecuente daño que se le puede continuar causando a la persona en lo político, en lo económico, en su honor, vida privada y/o imagen. Se afirma lo anterior, ya que para que se emita la sentencia en el recurso de apelación - es menester desahogar el procedimiento que está previsto en el ordena- miento legal antes citado y que a continuación abordaremos, tal como se lleva a cabo cuando se apela en contra de un auto o una sentencia que dicta un juez.

Luego de interpuesto el recurso en el acto de la notificación o a más tardar dentro de los tres días siguientes a que cause estado si fuere auto y cinco si fuere sentencia (artículo 241 del CFPC), el tribunal lo admite si procediere legalmente, y dentro de los tres días siguientes a la notificación lo remite al tribunal de apelación (artículo 242 del CFPC). A continuación se emplaza al apelante para que dentro de los tres días siguientes al estar notificado ocurra al tribunal a continuar la apelación (artículo 243 del CFPC), y en el escrito en el que se presente a continuar el recurso, se expresarán los agravios que le cause la resolución apelada y los conceptos por los que se hayan cometido (artículo 244 del CFPC). Recibidos los autos o el testimonio, el tribunal lo hace saber a las partes (artículo 245 del CFPC). Enseguida, en tres días examinará y declarará si el recurso fue interpuesto en tiempo, si es apelable la resolución, así como si el escrito del apelante fue presentado en tiempo y contiene expresión de agravios (artículo 246 del CFPC). Notifica a las partes para que puedan manifestar su disconformidad (artículo 250 del CFPC). Si admite la apelación, corre traslado a las demás partes, por el término de cinco días del escrito de expresión de agravios si se trata de sentencia, y tres si es un auto (artículo 252 del CFPC). En cuanto a las pruebas, se concederá un término para recibirlas de diez días (artículo 254 del CFPC). Se cita a las partes a la audiencia de alegatos, que se celebrará dentro de los diez días de fenecido el término del traslado; pero si se concedió término de prueba, quedará sin efecto la citación, y la audiencia se celebrará dentro de los diez días de concluido dicho término. El fallo se dictará dentro de los cinco días de verificada la audiencia (artículo 256 del CFPC). 


\begin{tabular}{|l} 
Recurso de APELACión \\
- Interposición del recurso: en la notificación o más tardar dentro de tres \\
días siguientes a que cause estado (auto), o cinco días siguientes a que \\
cause estado (sentencia). \\
- Remisión al tribunal de alzada: tres días siguientes a la notificación. \\
- Emplazamiento al apelante para que ocurra al tribunal a continuar el \\
- Análisis de la procedencia: tres días siguientes. \\
- - Audiencia: diez días del traslado. \\
Observación: si se concede término de prueba quedará sin efecto la ci- \\
tación, y la audiencia se celebrará dentro de los diez días de concluido \\
el término probatorio: \\
- Recepción de pruebas: diez días. \\
- Sentencia: cinco días de verificada la audiencia.
\end{tabular}

Obviamente, si el fallo no es favorable a quien ejerce el derecho de réplica, puede promover un amparo, con los tiempos que están fijados en la Ley de Amparo, ${ }^{1}$ tal como se señaló en el dictamen de la Cámara de Diputados: "no se descarta la posibilidad de que dichas sentencias... deriven en juicios de amparo".

En dicho dictamen se analizó lo relativo al procedimiento judicial. Tanto la diputada Valentina Batres Guadarrama como el diputado Alberto Amador Leal propusieron, cada uno por su lado, la procedencia del amparo para el caso en que el sujeto obligado se negará a publicar la réplica, pero únicamente se dijo: "Esta Comisión no coincide con que se considere, así sea de manera acotada, a los medios de comunicación como autoridad para los efectos de violentar derechos de los ciudadanos...". No obstante, no se exploró el nuevo concepto desarrollado por la Suprema Corte en el caso de amparo contra particulares. Tampoco se analizó a cabalidad la propuesta del diputado Jaime Cárdenas Gracia, quien estimó que "el procedimiento judicial federal propuesto es de una sola instancia ante un tribunal colegiado de circuito", ya que únicamente se dijo que se optaba por un juez de distrito y no por un tribunal colegiado de circuito, porque "no se desprende la existencia de actos administrativos". En tal virtud, considero que se debió profundizar más en la argumentación para refutar las propuestas de los legisladores mencionados.

1 La nueva Ley se publicó en el Diario Oficial de la Federación el 2 de abril de 2013.

2 Fue elaborado por la Comisión de Gobernación de la Cámara de Diputados el 5 de diciembre de 2013 y aprobado por el Pleno de la Cámara en la madrugada del día siguiente. 
Más aún, en el dictamen del Senado, ${ }^{3}$ en la parte medular, únicamente se dijo que se coincidía con la colegisladora respecto al imperativo de expedir una ley que regule los supuestos de procedencia del derecho de réplica, "así como los procedimientos que aseguren su cumplimiento...", y que también en los alcances de la minuta, por lo que estimo que hubo ausencia de fundamentación.

Como se puede observar de lo antes expuesto, en caso de que se llegue hasta el final del recurso de apelación y después se promueva el amparo y éste se sustancie hasta el dictado de la sentencia, por el tiempo que pase desde la publicación o transmisión de la información, pueden generarse - daños considerables al agraviado e incluso en alguna medida hasta a su - familia.

Aunado a lo anterior, conviene recordar que una de las iniciativas que se presentó en el Senado, concretamente la propuesta por Javier Corral Jurado, Marcela Torres Peimbert, Francisco García Cabeza de Vaca, Roberto Gil Zuarth, Víctor Hermosillo y Celada y Ernesto Ruffo Appel, proponía "diseñar un proceso de apelación expedito, para que, mediante un Magistrado del Tribunal Unitario de Circuito correspondiente, resuelva el fondo de la Litis... en un plazo de 13 días hábiles”. De esa manera se hubieran podido evitar las consecuencias negativas a que hicimos alusión para el solicitante del derecho de réplica.

\section{Conclusiones}

En México, quienes debieron reconocer el derecho de réplica en el ámbito constitucional y regularlo en el ámbito legal no lo hicieron puntualmente, pues tuvieron que pasar nueve décadas para que se estableciera en nuestra carta magna, y luego quince años más para que se expidiera la Ley Reglamentaria del Artículo 6o., párrafo primero, de la Constitución Política de los Estados Unidos Mexicanos, en materia del Derecho de Réplica.

En el desarrollo de este trabajo se analizaron los procedimientos extrajudicial y judicial que se deben sustanciar para hacer efectivo el derecho de réplica, y encontramos que en caso de que se llegue hasta la emisión de la sentencia en el juicio de amparo respectivo, por el lapso transcurrido desde la publicación o transmisión de la información "inexacta o falsa", puede perjudicarse considerablemente a las personas en alguno(s) de sus dere-

3 Fue elaborado por las Comisiones Unidas de Gobernación, de Justicia y de Estudios Legislativos Segunda de la Cámara de Senadores el 22 de septiembre de 2015 y aprobado por el Pleno de la Cámara el 13 de octubre de dicho año. 
Esta revista forma parte del acervo de la Biblioteca Jurídica Virtual del Instituto de Investigaciones Jurídicas de la UNAM

chos de la personalidad y traer repercusiones de tipo económico o político indeseables.

Cuando se discutió la Ley Reglamentaria del Artículo 6o., párrafo primero, de la Constitución Política de los Estados Unidos Mexicanos, en materia del Derecho de Réplica, en la Cámara de Diputados se debieron analizar a cabalidad los fundamentos que sustentaban las iniciativas presentadas en lo relativo a los plazos y términos contemplados en los procedimientos extrajudicial y judicial para hacer efectivo tal derecho. Por lo que respecta a la Cámara de Senadores, hubo una ausencia de argumentos de fondo, pues en esencia se dijo que se coincidía con los alcances del texto de la minuta de la colegisladora.

\section{BibliografíA}

BurgoA, Ignacio, 1982, Las garantías individuales, México, Porrúa.

Carbonell, Miguel, 2011, Los derechos fundamentales en México, México, Porrúa.

Chorny, Vladimir, 2015, "El derecho de réplica y su desastrosa reglamentación”, Derecho en acción, México, CIDE, disponible en: http://derecho enaccion.cide.edu/elderechodereplicaysudesastrosareglamental.

Cifuentes, Santos, 1995, Derechos personalísimos, Buenos Aires, Astrea.

Esteinou Madrid, Javier, 1994, "Derecho de réplica y medios electrónicos en México", Anuario de Investigación, México, UAM-X.

Ekmekdjian, Miguel A., 1996, Derecho a la información, Buenos Aires, Depalma.

Ghersi, Carlos A., 2000, Los nuevos daños-soluciones modernas de reparación, Buenos Aires, Hammurabi.

Islas, Jorge, 2002, "El derecho de réplica y la vida privada", en Alfonzo JiMÉNEZ, Armando (coord.), Responsabilidad social, autorregulación y legislación en radio y televisión, México, UNAM, Instituto de Investigaciones Jurídicas, disponible en: http://biblio.juridicas.unam.mx/libros/1/318/9.pdf.

Pierini, Alicia, 1998, Habeas data-derecho a la intimidad, Buenos Aires, Universidad.

Rosas Martínez, Alejandro, 2011, “¿Derecho de rectificación, derecho de respuesta o derecho de réplica?”, Derecho Comparado de la Información, México, núm. 18, julio-diciembre. 